\title{
Persistent posttraumatic cerebrospinal fluid leakage
}

\author{
Jonathan A. Friedman, M.D., Michael J. Ebersold, M.D., and Lynn M. Quast, R.N. \\ Department of Neurological Surgery, Mayo Clinic, Rochester, Minnesota
}

\begin{abstract}
Object. Persistent posttraumatic cerebrospinal fluid (CSF) leakage frequently complicates skull base fractures. Although many CSF leaks will cease without treatment, patients with CSF leaks that persist greater than 24 hours may be at increased risk for meningitis, and many will require surgical intervention. The authors reviewed their 15 -year experience with posttraumatic CSF leaks that persisted longer than 24 hours.

Methods. The authors reviewed the medical records of 51 patients treated between 1984 and 1998 with CSF leaks that persisted for 24 hours or longer after traumatic head injury. In 27 patients (55\%) spontaneous resolution of CSF leakage occurred at an average of 5 days posttrauma. In 23 patients $(45 \%)$ surgery was required to resolve the leakage. Eight patients $(16 \%)$ with occult CSF leaks presented with recurrent meningitis at an average of 6.5 years posttrauma. Forty-three (84\%) patients with CSF leaks sustained a skull fracture, most commonly involving the frontal sinus, whereas parenchymal brain injury or extraaxial hematoma was demonstrated in only 18 patients (35\%). Delayed CSF leaks, with an average onset of 13 days posttrauma, were observed in eight patients (16\%).

Among patients with clinically evident CSF leakage, the frequency of meningitis was $10 \%$ with antibiotic prophylaxis, and $21 \%$ without antibiotic prophylaxis. Thus, prophylactic antibiotic administration halved the risk of meningitis. A variety of surgical approaches was used, and no significant neurological morbidity occurred. Three (13\%) of 23 surgically treated patients required additional surgery to treat continued CSF leakage.

Conclusions. A significant proportion of patients with CSF leaks that persist greater than 24 hours will require surgical intervention. Prophylactic antibiotic therapy may be effective in this group of patients. Patients with skull base or frontal sinus fractures should be followed to detect the occurrence of delayed leakage. Surgery-related outcome is excellent.
\end{abstract}

\section{KEY WORDS • traumatic injury • cerebrospinal fluid fistula $・$ skull fracture}

Traumatic CSF leakage is a complication in $2 \%$ of all head-injured patients and in 12 to $30 \%$ of all cases of basilar skull fractures., ${ }^{4,13,14,17}$ Fractures involving the frontal or ethmoidal sinuses, and longitudinal temporal bone fractures, are most commonly associated with CSF leakage. ${ }^{17,21}$ Although penetrating head trauma is an obvious cause of both transient and persistent CSF fistulas, most traumatic CSF leaks occur as a result of blunt trauma, which is a result of the much higher incidence of blunt head trauma overall.

Most traumatic CSF leaks resolve spontaneously; the majority resolve within the first 24 to 48 hours. ${ }^{4,5,16}$ In fact, many leaks may go unrecognized or resolve so quickly, in the setting of the assessment and treatment of other more life-threatening injuries, that they go undocumented in the patient's permanent record. Furthermore, only persistent CSF fistulas become significant management challenges for the surgeon. Therefore, we focused our review on patients with posttraumatic CSF leakage persisting beyond 24 hours postinjury.

The mechanism of CSF leak cessation is thought to involve blood products and/or inflammatory adhesions at the site of the dural breach and associated skull fracture.

Abbreviations used in this paper: CSF = cerebrospinal fluid; $\mathrm{CT}=$ computerized tomography.
Herniation of brain tissue into the traumatic defect may also play a role in the cessation of the leak. However, persistent fistulas do occur, particularly in patients with fractures involving the anterior cranial fossa. The significance of a persistent CSF fistula is not the leak itself but its sequelae: posture-related headache, pneumocephalus, and most significantly, bacterial meningitis. Overall, between $7 \%$ and $30 \%$ of all patients with posttraumatic CSF leakage will develop meningitis, and this rate increases as the duration of CSF leakage increases., ${ }^{42,14,15,18,24}$ The role of antibiotic prophylaxis in patients with CSF leaks has been studied extensively yet remains controversial. However, because patients with persistent leaks are at higher risk of meningitis, the efficacy of antibiotic prophylaxis may be higher in this subgroup.

The appropriate timing for surgical intervention in patients with persistent posttraumatic CSF leaks is also unclear. What is clear is that many surgical approaches, both intracranial and extracranial, can be successful, depending on patient factors and the anatomy of the fistula. We reviewed our single-institution experience over a 15-year period with patients in whom traumatic CSF leaks persisted longer than 24 hours.

\section{CLINICAL MATERIAL AND METHODS}

We reviewed the medical records and CT studies ob- 
J. A. Friedman, M. J. Ebersold, and L. M. Quast
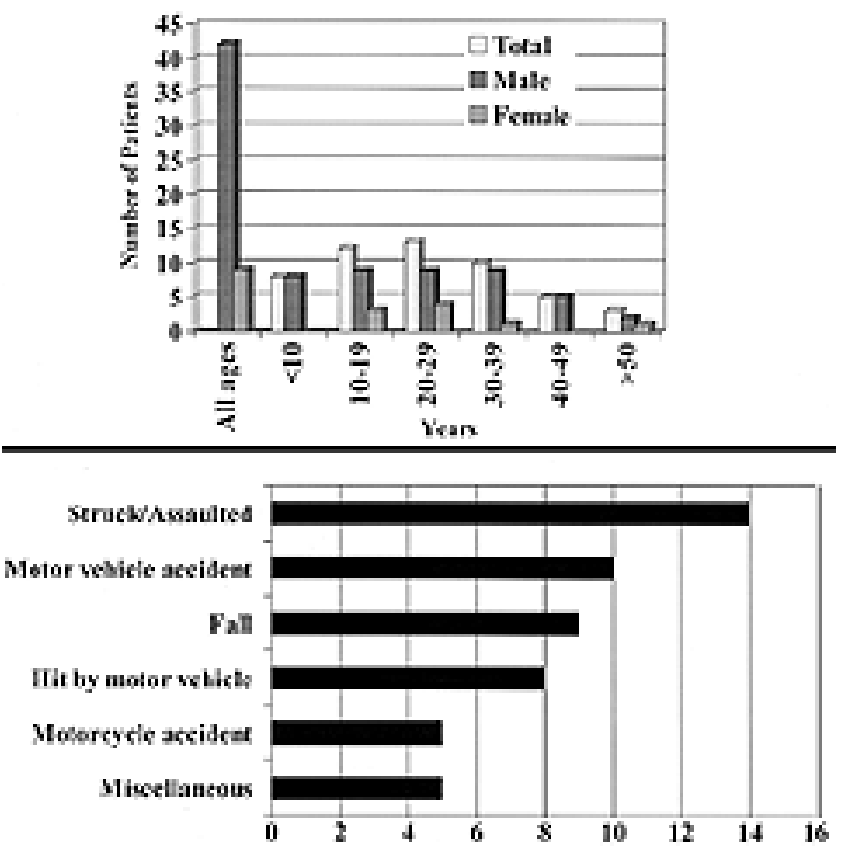

Fig. 1. Upper: Bar graph showing patient age and sex. Lower: Graph highlighting the mechanism of injury.

tained in 101 patients in whom traumatic CSF leaks had been diagnosed and treated at Mayo Clinic between 1984 and 1998. In 51 patients, CSF leak was present 24 hours or longer postinjury. The remaining 50 patients had CSF leaks that resolved within 24 hours, spinal leaks, remote history of leak, or uncertain clinical evidence for a true CSF leak. These patients were excluded from analysis.

Data were collected regarding patient demographics, mechanism of injury, clinical presentation, CT findings, antibiotic prophylactic agents, development of meningitis, and timing and outcome of surgery. Referral source was also noted, when applicable, to determine whether there was a possible source of bias.

\section{RESULTS}

The age and male/female ratio are depicted in Fig. 1 upper. As expected in a trauma series, young males are highly represented. The mechanisms of injury in our 51 cases are shown in Fig. 1 lower. Only one case of penetrating head trauma was noted, which exemplifies the overall low incidence of this injury in our population.

In $43(84 \%)$ of 51 patients we observed associated skull fractures on noncontrast head CT scans. The most common fracture associated with CSF leakage was that of the frontal bone involving the frontal sinus, followed by orbital and petrous fractures. In $18(35 \%)$ of 51 patients CT scanning revealed parenchymal brain injury or extraaxial hematoma. Although many patients underwent either a radioisotope study or CT scanning with metrizamide cisternography that demonstrated the CSF leak, the clinical findings were diagnostic in most cases. Rhinorrhea was the primary clinical manifestation in 22 patients $(43 \%)$, whereas otorrhea was the primary finding in 17 patients $(33 \%)$. One patient experienced both rhinorrhea and otor-

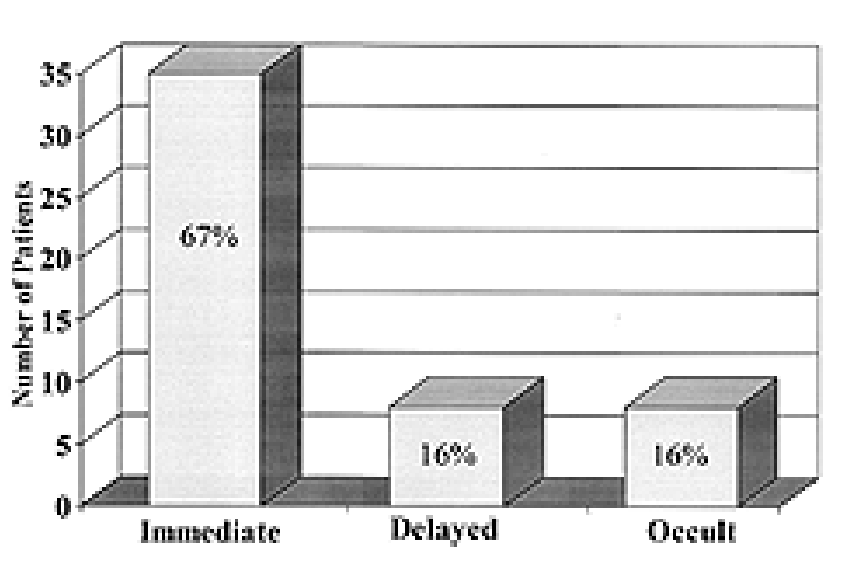

Fig. 2. Bar graph classifying timing from traumatic event to onset of CSF leak.

rhea. In one patient the CSF leaked down the back of the throat only. In one patient the CSF drained through a scalp laceration overlying an occipital fracture. One patient presented with tension pneumocephalus. Of particular interest are the eight patients (16\%) in whom clinically occult leaks were found and who presented with recurrent bacterial meningitis: in these patients, CSF fistulas were demonstrated by radioisotope or metrizamide-enhanced CT cisternography (Fig. 2). These patients presented at an average of 6 years posttrauma and clearly represent a distinct subgroup in terms of risk for meningitis and use of antibiotic prophylactic agents.

Eight patients presented with delayed CSF leaks; that is, the leakage was clinically evident but began at some time interval after the trauma (Fig. 2). In the patients with delayed CSF leaks, the average time interval between the trauma and the leakage was 13 days (range 1-30 days; Fig. 3). In three conservatively treated patients who developed recurrent leakage after initial cessation, surgery was required.

The overall frequency of meningitis in this series was $27.5 \%$. This includes the eight patients with occult CSF leaks who presented with meningitis. Of the 43 patients with clinically evident leaks, six (14\%) suffered at least one episode of meningitis (Fig. 4 upper). Streptococcus pneumoniae was by far the most common pathogen. Two patients developed fungal brain abscesses, with either preceding or concomitant meningitis. There were no cases of meningitis-associated death in this study. The incidence of meningitis-related morbidity was also quite low: one patient developed a seizure disorder, and another patient sustained mild cognitive dysfunction. Of the 43 patients with clinically evident leaks, 29 were treated with antibiotic prophylactic agents, whereas 14 received none. Three $(10 \%)$ of the patients treated with antibiotic prophylaxis developed meningitis, as did three $(21 \%)$ of the patients who did not receive prophylaxis (Fig. 4 upper). This difference was not statistically significant $(\mathrm{p}=0.33)$.

In $27(53 \%)$ of 51 patients spontaneous resolution of the CSF leaks occurred at an average of 4.8 days (Fig. 4 lower). Spontaneous resolution of the CSF leak ocurred in $26(60 \%)$ of the 43 patients with clinically evident leaks. Patients with rhinorrhea were far more likely than those with otorrhea to require surgery for leak cessation: $45 \%$ of 


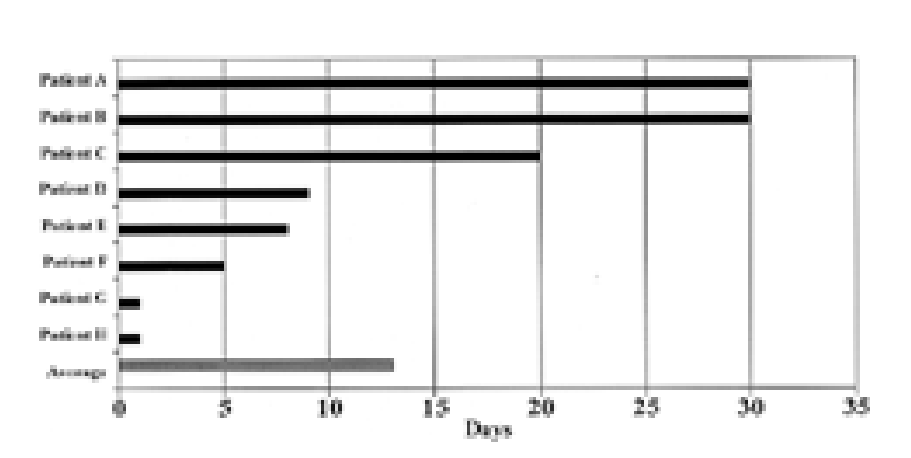

Fig. 3. Bar graph showing the time interval from trauma to onset of CSF leak in patients with delayed leaks.

patients with rhinorrhea ultimately required surgery, compared with only $12 \%$ of those with otorrhea. Patients who presented with clinically occult CSF leaks were generally treated surgically once the site of the leakage was identified: seven of these eight patients underwent surgery whereas spontaneous cessation of the leakage occurred in only one (Fig. 4 lower).

The median time interval from initial traumatic event to surgery for all patients was 45 days. For patients with clinically evident leaks, this median time interval was 16 days. The time to surgery was markedly higher for those patients with occult leaks who presented with recurrent meningitis (median time to surgery 6 years).

Twenty-four patients $(47 \%)$ were treated surgically. A variety of surgical approaches was used: most were intracranial but some extracranial and combined intracranialextracranial procedures were performed. There were no deaths or neurological deficits as a result of surgical treatment. Two patients developed wound infections that were successfully treated with antibiotic medication, and surgical revision was not required. Of the 24 surgically treated patients, three $(12.5 \%)$ experienced further CSF leakage requiring reoperation. One of these patients developed meningitis and a brain abscess postoperatively, which ultimately required surgical drainage. No patient suffered ongoing or recurrent leakage after reoperation.

Thirty-five of 43 patients with clinically evident CSF leaks presented from the primary trauma catchment of our institution, whereas the remaining eight patients with clinically evident CSF leaks were referred from other hospitals. Six of eight patients with occult CSF leaks were initially evaluated at other hospitals and were then referred to our institution for further care.

\section{DISCUSSION}

The rate of spontaneous cessation of traumatic CSF leaks has been reported to be 80 to $95 \% .^{4,16}$ In our series, the rate of spontaneous cessation was only $53 \%$, far less than that reported in other studies. Even after excluding cases of occult CSF leaks, the rate of spontaneous cessation of clinical CSF leakage was only $60 \%$. This low rate of spontaneous cessation cannot be explained by premature operative repair because the mean time to operation of 16 days represents a conservative management approach. In many series of traumatic CSF leakage the investigators include patients with leaks that resolved with- in the first 24 hours, which ours did not. ${ }^{4,12,13}$ Furthermore, reports of traumatic basilar skull fractures are selective for CSF otorrhea, which is more likely to resolve spontaneously. ${ }^{4,11,16}$ Analysis of our findings suggests that when all cases of traumatic CSF leaks are considered, those that persist greater than 24 hours have a relatively high rate of requiring operative repair.

Some authors have suggested that lumbar drainage may be safe and effective treatment in patients with traumatic CSF leakage. ${ }^{23}$ Lumbar drainage was used in only a few patients in this series, and no meaningful conclusions could be drawn regarding its efficacy. It is possible, however, that the rate of surgical repair may have been lower had we undertaken more aggressive implementation of lumbar drainage.

Referral bias did not have a significant effect on overall results. In the 43 patients with clinically apparent CSF leaks, eight were referred from other institutions. Whereas there was a higher likelihood (five of eight) that these eight patients would require surgery, even when these patients are excluded, $34 \%$ of the patients with clinically evident CSF leaks ultimately were treated surgically. However, a significant referral bias was present with regard to the patients with occult CSF leaks: six of eight patients with occult leaks were referred from outside institutions.

Cerebrospinal fluid leaks due to fractures involving the anterior cranial fossa were much more likely to require

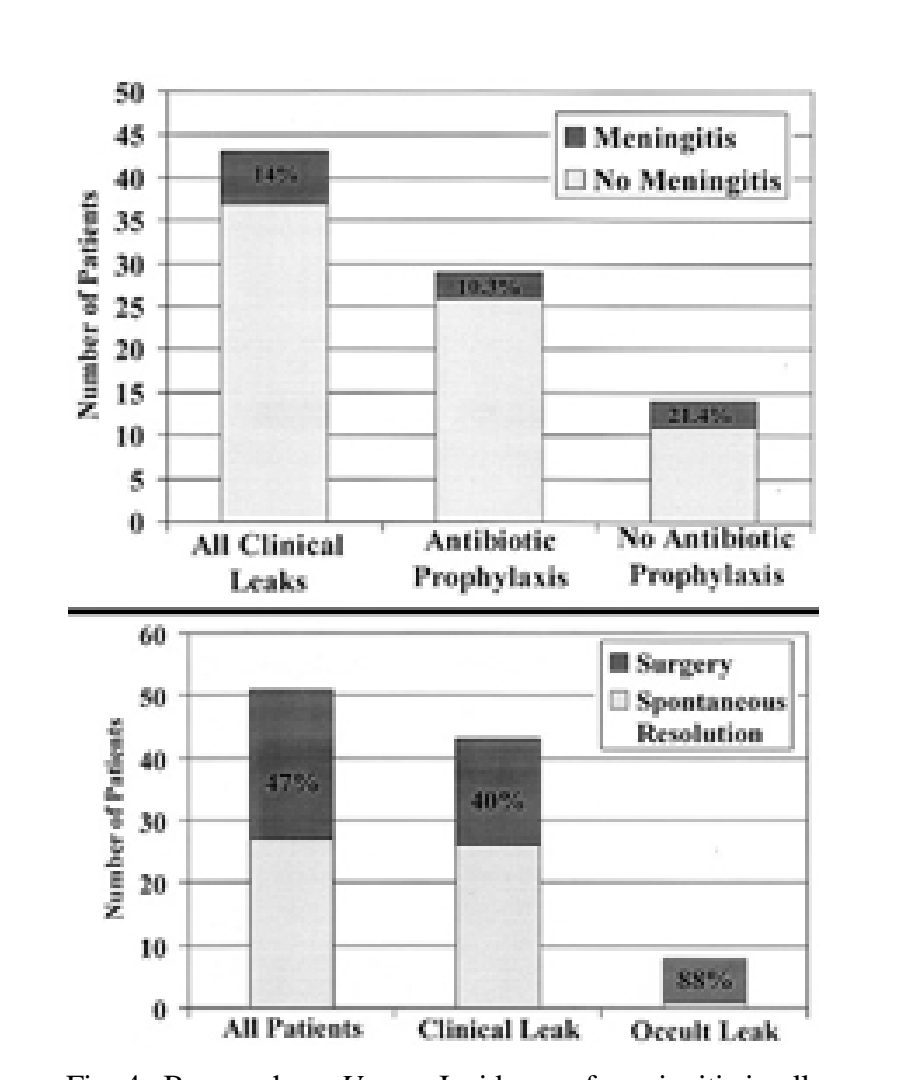

Fig. 4. Bar graphs. Upper: Incidence of meningitis in all patients with clinically evident CSF leaks, treated with and without antibiotic prophylactic medication. Lower: Rate of spontaneous resolution compared with surgical intervention in all patients, patients with clinically evident CSF leaks, and patients with occult CSF leaks. 


\section{J. A. Friedman, M. J. Ebersold, and L. M. Quast}

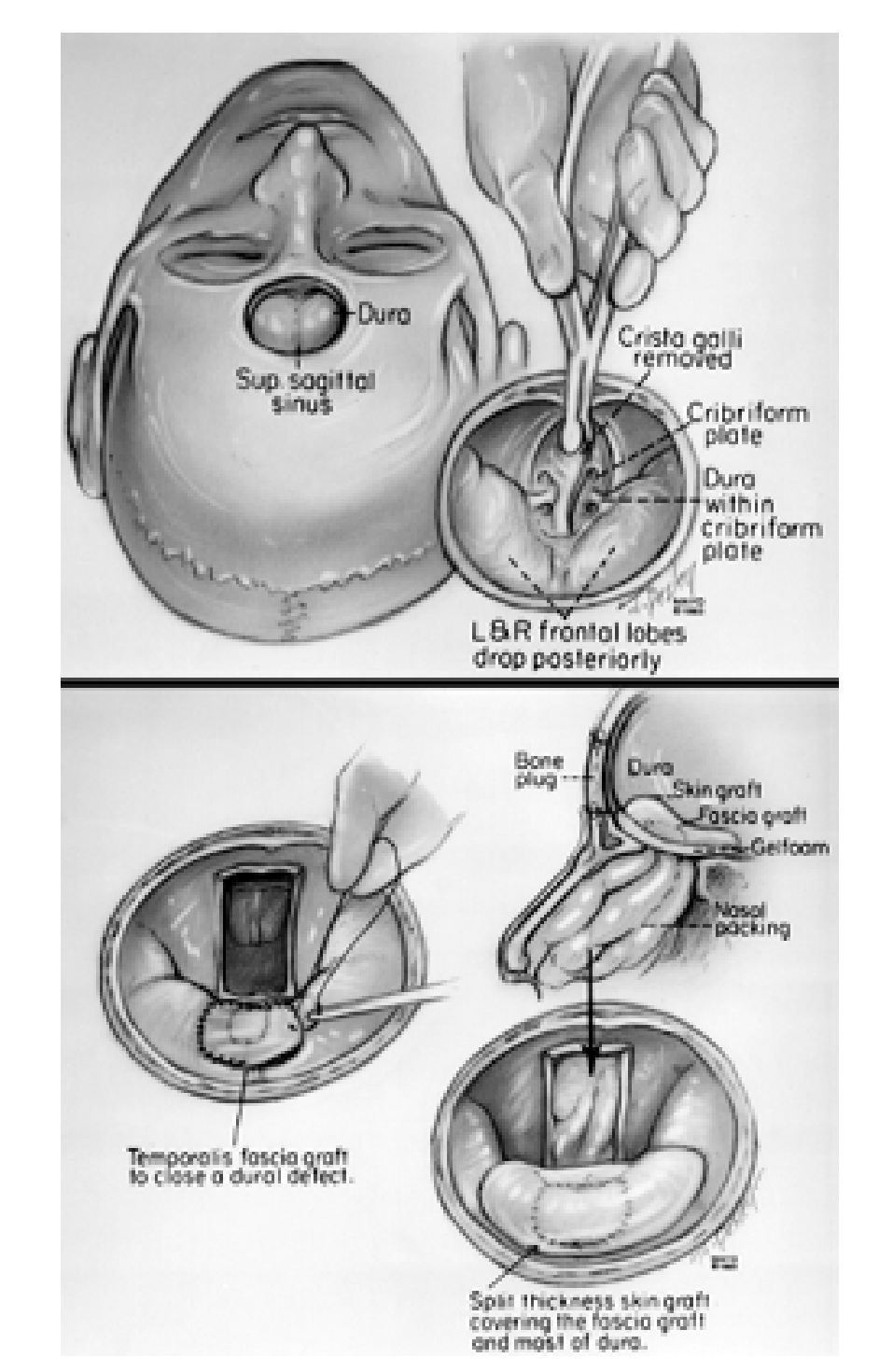

Fig. 5. Artist's drawings. Upper: A midline, low-frontal trephine craniotomy for an extradural approach to the anterior cranial fossa floor is shown. Fractured fragments of the crista galli and cribiform plate are removed. Lower: The dural defect is closed in water-tight fashion with temporalis fascia, then buttressed with a split-thickness skin graft that is tacked to the dura. Gelfoam and fibrin glue line the floor of the anterior fossa extradurally. In this illustration, extensive removal of a fractured cribiform plate has left a substantial defect, and there is communication of the intracranial space and the nasal cavity. The nose has been packed from below. sup. $=$ superior; $\mathrm{L} \& \mathrm{R}=$ left and right.

surgical repair than those caused by temporal bone fractures. Consequently, $45 \%$ of patients with CSF rhinorrhea required surgery compared with only $12 \%$ of those with CSF otorrhea. Cerebrospinal fluid leaks associated with temporal bone fractures have historically been thought to have a higher propensity to cease spontaneously. Indeed, in a large series of temporal bone fractures, CSF leaks in only five of 122 patients persisted longer than 14 days. ${ }^{3}$

Eight patients presented with delayed CSF leaks. The average time interval was 13 days but ranged up to 30 days. Other investigators have reported a similar inci- dence of delayed posttraumatic CSF leakage. ${ }^{9,14,20}$ Furthermore, three patients who developed recurrent leakage after initial cessation required surgery. The implication of the delayed leakage is that patients should be given explicit instruction regarding surveillance for CSF leakage and that short-term follow-up observation is indicated.

The overall incidence of meningitis in our series was $27.5 \%$. This is relatively high in comparison with other published reports. ${ }^{6,7,11,16}$ However, when only patients with clinically evident CSF leaks are considered, the meningitis rate was $14 \%$, a rate more in keeping with the literature. Surprisingly, $15 \%$ of patients presented with recurrent meningitis from occult posttraumatic CSF leakage. Although it is likely that some degree of referral bias explains this high representation of patients with occult leaks in our series, clearly patients presenting in this condition represent a significant subgroup of posttraumatic CSF leaks. We were unable to demonstrate that a longer duration of CSF leakage was associated with an increased frequency of meningitis, although this has been suggested by others. ${ }^{12,18,24}$ That pneumococcus was the most frequent pathogen in our patients is not surprising. $2,8,10$

The efficacy of prophylactic antibiotic agents in the setting of posttraumatic CSF leakage is controversial. The contrasting findings reported by two large studies in which metaanalysis was conducted reflect the general disagreement in the literature. ${ }^{3,25}$ One possible reason for the inability of studies to demonstrate the efficacy of antibiotic prophylaxis is the low incidence of meningitis, which requires a large number of patients to have sufficient power. In our study, a trend shown toward the efficacy of antibiotic prophylaxis - the use of antibiotic agents halved the incidence of meningitis - but we did not have a sufficient number of cases to attain statistical significance. In most studies on the use of antibiotic prophylactic agents in traumatic CSF leakage the authors have considered all patients with traumatic CSF leaks, including the many patients in whom the leaks spontaneously subside within the first 24 hours. These are the patients who would theoretically benefit least from prophylaxis, given the short duration of leakage. It is possible that the subset of patients with persistent CSF leaks may indeed benefit from the use of antibiotic prophylactic agents.

A variety of surgical approaches can be used to obtain successful outcome, but the selection of an approach must be based on individual patient factors and, most importantly, the anatomy of the CSF fistula. Traditionally, anterior fossa CSF leaks are approached intracranially via a bifrontal craniotomy, providing excellent exposure of the floor of the anterior cranial fossa for both intradural and extradural approaches. However, we frequently use a midline frontal trephine craniotomy, which is smaller and typically provides adequate access to approach the anterior skull base extradurally (Fig. 5). In the event of infection and loss of the bone plate, the defect from the trephine is significantly less substantial than that caused by the bifrontal craniotomy, and cosmetic repair is therefore easier. After the craniotomy is performed, loose bone fragments are removed. The dural tear is identified and closed primarily, if possible. This may or may not require modest resection of herniated brain tissue. Sometimes the dural tear is extensive and a graft must be used, and an autologous graft, such as fascia lata or pericranium, is preferable 
to bovine pericardium or other nonautologous material given the contaminated nature of the field. Frequently, fibin glue is used as a sealant, although its true efficacy in preventing CSF fistulas is unproven.

In most cases one or more sinuses is involved in the fracture and, consequently, the fistula. Obliteration of these sinuses is crucial not only in stopping the CSF fistula but also in preventing mucocele and infection. For fractures involving the frontal sinus, this is accomplished first by meticulous stripping of sinus mucosa and then by packing with fat or muscle. Removal of the posterior table of the frontal sinus is an effective means of "cranializing" the sinus and is useful in some circumstances. For small ethmoidal fractures, external ethmoidectomy, exenteration of all ethmoidal air cells, and obliteration of the dural defect with muscle or fat packing is appropriate. We generally use a lateral rhinotomy for this extracranial approach. Endoscopic approaches have been described and shown to be effective in certain circumstances, although we have not used these routinely. ${ }^{1,19,22}$ The sphenoid sinus and cribriform plate region can be approached via the nasal cavity, or translabially in the case of the sphenoid, with mucosal stripping and packing with fat or muscle; a routine transsphenoidal approach allows good visualization of this region.

For defects of the middle cranial fossa, an intracranial approach via a temporal craniotomy and primary dural closure, or an extracranial approach via a mastoidectomy, can be used. The extracranial approach provides the advantage of allowing for extensive packing of the middle ear with fat and cartilage, but it may be contraindicated if hearing is preserved.

Overall surgical results were quite satisfactory, with no mortality or neurological morbidity. There were two wound infections that did not require reoperation. Three of 24 surgically treated patients required reoperation for recurrent leakage: the second operation successfully resolved the leakage in all cases, although one patient did develop pneumocephalus and a brain abscess prior to the second operation. Surgical intervention is appropriate in patients with ongoing CSF leaks and/or those who develop meningitis.

\section{References}

1. Anand VK, Murali RK, Glasgold MJ: Surgical decisions in the management of cerebrospinal fluid rhinorrhea. Rhinology 33: 212-218, 1995

2. Appelbaum E: Meningitis following trauma to the head and face. JAMA 173:1818-1822, 1960

3. Brodie H: Prophylactic antibiotics for posttraumatic cerebrospinal fluid fistulae. A meta-analysis. Arch Otolaryngol Head Neck Surg 123:749-752, 1997

4. Brodie H, Thompson TC: Management of complications from 820 temporal bone fractures. Am J Otol 18:188-197, 1997

5. Chandler JR: Traumatic cerebrospinal fluid leakage. Otolaryngol Clin North Am 16:623-632, 1983

6. Choi D, Spann R: Traumatic cerebrospinal fluid leakage: risk factors and the use of prophylactic antibiotics. Br J Neurosurg 10:571-575, 1996

7. Clemenza JW, Kaltman SI, Diamond DL: Craniofacial trauma and cerebrospinal fluid leakage: a retrospective clinical study. $\mathbf{J}$ Oral Maxillofac Surg 53:1004-1007, 1995

8. Frazee R, Mucha P Jr, Farnell MB, et al: Meningitis after basilar skull fracture. Does antibiotic prophylaxis help? Postgrad Med 83:267-268, 273-274, 1988

9. Jefferson A, Reilly G: Fractures of the floor of the anterior cranial fossa. The selection of patients for dural repair. Br J Surg 59:585-592, 1972

10. Kaufman BA, Tunkel AR, Pryor JC, et al: Meningitis in the neurosurgical patient. Infect Dis Clin North Am 4:677-701, 1990

11. Lee D, Honrado C, Har-El G, et al: Pediatric temporal bone fractures. Laryngoscope 108:816-821, 1998

12. Leech PJ, Paterson A: Conservative and operative management of cerebrospinal-fluid leakage after closed head injury. Lancet 1:1013-1016, 1973

13. Lewin W: Cerebrospinal fluid rhinorrhea in closed head injuries. Br J Surg 42:1-18, 1954

14. Lewin W: Cerebrospinal fluid rhinorrhea in nonmissile head injuries. Clin Neurosurg 12:237-252, 1966

15. MacGee E, Cauthen JC, Brackett CE: Meningitis following acute traumatic cerebrospinal fluid fistula. J Neurosurg 33: 312-316, 1970

16. McGuirt WF Jr, Stool SE: Cerebrospinal fluid fistula: the identification and management in pediatric temporal bone fractures. Laryngoscope 105:359-364, 1995

17. Mendizabal GR, Moreno BC, Flores CC: Cerebrospinal fluid fistula: frequency in head injuries. Rev Laryngol Otol Rhinol 113:423-425, 1992

18. Mincy J: Post-traumatic cerebrospinal fluid fistula of the frontal fossa. J Trauma 6:618-622, 1966

19. Ng M, Maceri DR, Levy MM, et al: Extracranial repair of pediatric traumatic cerebrospinal fluid rhinorrhea. Arch Otolaryngol Head Neck Surg 124:1125-1130, 1998

20. Okada J, Tsuda T, Takasugi S, et al: Unusually late onset of cerebrospinal fluid rhinorrhea after head trauma. Surg Neurol 35: 213-217, 1991

21. Ommaya A: Cerebrospinal fluid fistula and pneumocephalus, in Wilkins RH, Rengachary SS (eds): Neurosurgery. New York: McGraw-Hill, 1996, Vol 2, pp 2773-2782

22. Sethi D, Chan C, Pillay PK: Endoscopic management of cerebrospinal fluid fistulae and traumatic cephalocele. Ann Acad Med 25:724-727, 1996

23. Shapiro SA, Scully T: Closed continuous drainage of cerebrospinal fluid via a lumbar subarachnoid catheter for treatment or prevention of cranial/spinal cerebrospinal fluid fistula. Neurosurgery 30:241-245, 1992

24. Spetzler RF, Wilson CB: Management of recurrent CSF rhinorrhea of the middle and posterior fossa. J Neurosurg 49: 393-397, 1978

25. Villalobos T, Arango C, Kubilis P, et al: Antibiotic prophylaxis after basilar skull fractures: a meta-analysis. Clin Infect Dis 27:364-369, 1998

Manuscript received May 11, 2000.

Accepted in final form June 20, 2000.

Address reprint requests to: Jonathan A. Friedman, M.D., Department of Neurological Surgery, Joseph 1-229, Saint Mary's Hospital, 1216 Second Street S.W., Rochester, Minnesota 55905. email: friedman.jonathan@mayo.edu. 